where $\Omega$ is a constant, where $a(t), b(t), c(t)$ are arbitrary functions of $t$, and where

$$
\begin{aligned}
& Y=(y-b) \cos \Omega t+(z-c) \sin \Omega t \\
& Z=-(y-b) \sin \Omega t+(z-c) \cos \Omega t .
\end{aligned}
$$

Comparison of (3) with (8) makes quite evident that these two solutions are essentially different. Whereas (8) is exact, (3) is approximate. Furthermore, (3) cannot be derived as an approximation from (8).

\title{
A MEASURE OF THE AREA OF A HOMOGENEOUS RANDOM SURFACE IN SPACE*
}

\section{By STANLEY CORRSIN (Aeronautics Department, The Johns Hopkins University)}

Introduction. We are given an indefinitely large space containing random surface or surfaces homogeneously located in the mean. The problem is to relate the average area of surface per unit volume of space to a simpler statistical quantity, in particular the average number of cuts per unit length made by a straight randomly directed sampling line with the surface.

The plane case will be studied first. After the three dimensional case, illustrative application will be made to the problem of extending to two and three dimensional variables a theorem of $\mathrm{S}$. O. Rice on the average rate of occurrence of any particular value of a one dimensional random variable. Possible use in describing fluid mixing is also indicated.

Two dimensions. Given a plane "homogeneously" inscribed with contour or contours of arbitrary shape. The homogeneity is statistical, i.e. any statistical function associated with the contours is invariant to a translation of coordinate system in the plane. Let $\&$ be the average contour length enclosed in unit area and let $n$ be the average number of cuts per unit length made by an arbitrary straight traverse line crossing the plane. For a non-isotropic field $n$ is averaged over all traverse directions with uniform weighting; for an isotropic field, any single line will do.

Draw a "very large" square in the plane, $L$ on a side, and subdivide it into "very narrow" traverse strips parallel to one pair of sides.

"Very large" here denotes $L$ so large that averages over $L$ or $L^{2}$ are satisfactorily close to their asymptotic values. For example it requires that each traverse strip cross the contours a very large number of times and that the length of contour in $L^{2}$ divided by $L^{2}$ be as close as we like to $\mathcal{L}$. "Very narrow" denotes $\delta$ so small that virtually all of the intercepted contour segments in a strip can be approximated by secants. This gives restriction on the permissible number of corners and contour intersections.

We imagine the square and strip structure rotated through $180^{\circ}$ for averaging purposes in case the field is not isotropic. Then the average number of crossings in one

*Teceived Jan. 18, 1954. Supported by the Office of Naval Research. 
strip is $n L$. The number of strips is $L / \delta$. Hence the expected total contour length in the square is

$$
\mathfrak{L}_{L^{2}}=\frac{n L^{2}}{\delta}\langle l\rangle,
$$

where $\langle l\rangle$ is the average contour segment per crossing, so (1) can be written

$$
\mathfrak{L}=\frac{n}{\delta}\langle l\rangle .
$$

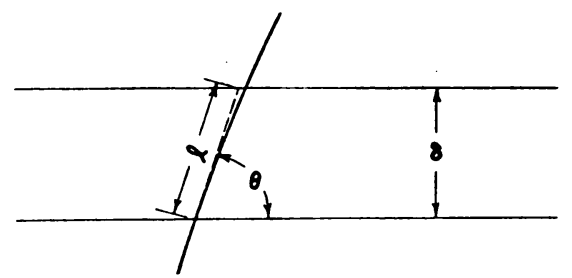

Fig. 1

To obtain $\langle l\rangle$, consider a typical crossing (Fig. 1). For any one crossing

$$
l=\frac{\delta}{|\sin \theta|} .
$$

However, for each traverse orientation, the probability of intersection at angle $\theta$ with a contour element is proportional to the projected length of the element on a line perpendicular to the traverse direction, i.e. to $|\sin \theta|$. With normalization this becomes the probability density of

$$
\beta_{\theta}(\theta)= \begin{cases}\frac{1}{2}|\sin \theta| & -\frac{\pi}{2} \leq \theta \leq \frac{\pi}{2} \\ 0 & \text { elsewhere. }\end{cases}
$$

But

$$
\langle l\rangle=\int_{0}^{\infty} l \beta_{l}(l) d l=2 \int_{0}^{\pi / 2} l(\theta) \beta_{\theta}(\theta) d \theta .
$$

Substituting (4) into (5) we find

$$
\langle l\rangle=\frac{\pi}{2} \delta,
$$

whence

$$
n=\frac{2}{\pi} \mathfrak{L} .
$$

This result is easily seen to be consistent with the solution of Buffon's "needle problem" $\dagger$

*Arrived at independently by P. V. Danckwerts of Cambridge University (private communication). tSee, for example, Uspensky: Introduction to mathematical probabilit!, McGraw-Hill Book ('o., 1937. 
in the special case when the contours are efuidistant parallel straight lines.

For an isotropic field $\&$ can thus be determined by the intersection rate along a single sampling traverse.

Three dimensions. Low wave a three dimensional space homogeneously inscribed with surface or surfaces of arbitrary shape. Define $a$ as the average surface area per unit volume of space and $n$ as the average number of cuts per unit length made by a random straight line traverse across the space. For a nonisotropic field $n$ is averaged over all directions with equal weight; for an isotropic field a single traverse suffices.

Take a "very large" cube ( $L$ on a side) and subdivide into "very narrow" square traverse tubes $(\delta \times \delta \times L)$ with all faces parallel to those of the cube.

The average number of surface crossings in a single tube is $n L$. The number of tubes in $L^{3}$ is $L^{2} / \delta^{2}$. Hence the expected surface area in the cube is

$$
\alpha_{L^{3}}=n L \frac{L^{2}}{\delta^{2}}\langle a\rangle,
$$

where $\langle a\rangle$ is the average area segment per crossing. $Q_{L^{3}}=a \cdot L^{3}$, so (8) can be written

$$
a=\frac{n}{\delta^{2}}\langle a\rangle .
$$

To obtain $\langle a\rangle$, consider a typical crossing (Fig. 2), with $\varphi$ the angle between tube axis and normal to surface element.

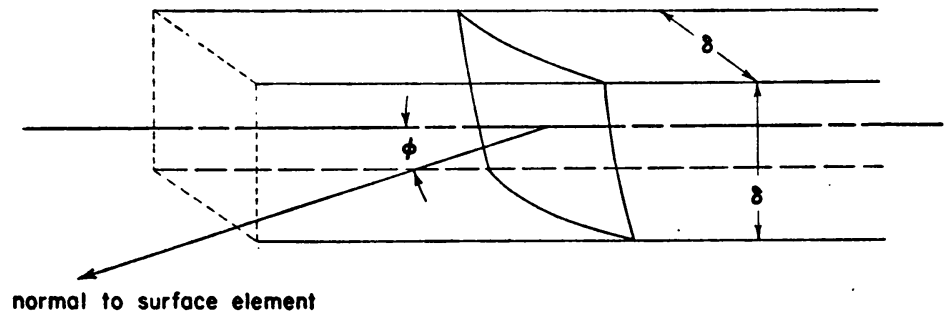

FIG. 2

$$
a=\frac{\delta^{2}}{|\cos \varphi|} .
$$

For each traverse orientation (i.e. cube orientation) the probability of intersection at angle $\varphi$ with a surface element is proportional to the projected area of the surface on a plane perpendicular to the tube axis, i.e. to $|\cos \varphi|$ times a measure of relative solid angle giving $\varphi$, i.e. $|\sin \varphi|$. With normalization constant, this gives the probability density of $\varphi$

$$
\beta_{\varphi}(\varphi)= \begin{cases}2|\cos \varphi| \cdot|\sin \varphi| & 0 \leq \varphi \leq \frac{\pi}{2} \\ 0 & \text { elsewhere }\end{cases}
$$

therefore

$$
\langle a\rangle=\int_{0}^{\infty} a \beta_{a}(a) d a=\int_{0}^{\pi / 2} a(\varphi) \beta_{\varphi}(\varphi) d \varphi .
$$


Substituting (10) and (11) into (12), we find

$$
\langle a\rangle=2 \delta^{2} .
$$

Whence

$$
n=\frac{a}{2} .
$$

Again, for an isotropic field, $a$ can be determined by the intersection rate along a single sampling traverse.

\section{Application I: Rice's theorem in two and three dimensions.}

Given a two dimensional stationary ( $\equiv$ homogeneous) isotropic random variable $u(x, y)$. The iso-value lines corresponding to $u=u_{c}$ will be an isotropic field of contours in the plane, and we can seek the average occurrence length per unit area of the value $u_{c}$ :

$$
\mathcal{L}_{u_{c}}=\frac{\pi}{2} n_{u_{c}},
$$

where $n_{u_{c}}$ is the average number of $u_{c}$ occurrences per unit length along the linear sampling traverse in any direction. The traverse yields a one dimensional random variable to which we can apply a theorem of Rice:*

$$
n_{u_{c}}=\int_{-\infty}^{\infty} \gamma\left(u_{c}, u^{\prime}\right)\left|u^{\prime}\right| d u^{\prime},
$$

where $u^{\prime} \equiv \partial u / \partial s$, the derivative in the direction of our sampling traverse and $\gamma\left(u, u^{\prime}\right)$ is the joint probability density of $u$ and its slope in this sample. From (15) and (16)

$$
\mathfrak{L}_{u_{c}}=\frac{\pi}{2} \int_{-\infty}^{\infty} \gamma\left(u_{c}, u^{\prime}\right)\left|u^{\prime}\right| d u^{\prime} .
$$

In the special case of jointly Gaussian $u$ and $u^{\prime}$, we use Rice's simplified result to obtain

$$
\mathscr{L}_{u_{c}}=\frac{1}{4}\left\{-\frac{\Psi^{\prime \prime}(0)}{\Psi(0)}\right\}^{1 / 2} e^{-u^{2} / 2 \Psi(0)}
$$

where $\Psi(\sigma)$ is the auto-correlation, $\langle u(s) u(s+\sigma)\rangle$.

For three dimensions, the corresponding equations are

$$
a_{u_{c}}=2 \int_{-\infty}^{\infty} \gamma\left(u_{c}, u^{\prime}\right)\left|u^{\prime}\right| d u^{\prime}
$$

and, for jointly Gaussian $u$ and $u^{\prime}$,

$$
a_{u_{c}}=\frac{1}{\pi}\left\{-\frac{\Psi^{\prime \prime}(0)}{\Psi(0)}\right\}^{1 / 2} e^{-u^{2 / 2 \Psi(0)}} .
$$

For non-isotropic, homogeneous $u(x, y), \gamma\left(u, u^{\prime}\right)$ is a function of traverse direction, and we must include the operation of averaging over all directions.

*S. O. Rice, Mathematical analysis of random noise, Bell System Tech. J., 23 (3) and 24 (1), July 1944 and January 1945. 


\section{A pplication II: Fluid mixing.}

The average interfacial area per unit volume is a significant measure of the "degree of mixedness" in an isotropic field of two molecularly immiscible liquids. It is, however, inaccessible to straightforward experimental determination. Equation (14) permits its calculation from the simpler process of interfacial-intersection counting along a linear traverse through the mixture.

In the homogeneous mixing of two gases or molecularly miscible liquids (e.g. turbulent mixing) it is possible that the notion of interfacial area can be replaced either by the surface area on which the concentration fluctuation is zero, or by the surface on which the concentration gradient magnitude has a local maximum.

\section{EQUILIBRIUM OF MEMBRANES ELASTICALLY SUPPORTED AT THE EDGES*}

\section{BY V. (i. HART (Dublin Institute for Advanced Studies)}

Abstract. The problem considered is that of finding the statical deflection of a stretched membrane, subjected to a uniform pressure on one side and elastically supported at the edges. The deflection of the membrane is supposed small and the problem reduces to solving Laplace's equation with mixed boundary conditions. Solutions are given for the cases where the bounding curve of the membrane is (i) an equilateral triangle, and (ii) a rectangle.

1. Introduction. We consider the problem of finding the statical deflection of a membrane originally lying in a plane (the neutral plane), when subjected to a uniform pressure on one side, its edge being elastically supported. This means that the edge can move in a direction perpendicular to the neutral plane, but is restrained at any point by a force proportional to the deflection at that point. Small deflections only being considered, the tension is a constant, and the problem reduces to solving the boundaryvalue problem (3) for an edge of arbitrary shape; solutions are given for (i) a membrane in the form of an equilateral triangle and (ii) a rectangular membrane.

Imagine a membrane stretched to a uniform tension $T$ and bounded by any plane curve $B$. A uniform pressure $P$ now acts on one side of the membrane which takes up a statical deflected position with deflection $w$. At any point on the edge the tension gives a component of force per unit length in the direction perpendicular to the neutral plane of amount $-T \partial w / \partial n$, ( $\partial n$ being the outward normal element to $B$ in the neutral plane), and this is balanced by the elastic force of constraint, which we write $w / k$, where $k$ is a constant.

The appropriate partial differential equation and boundary condition for $w$ are therefore

$$
\Delta w=-P / T \text { in } S, \quad(w)_{B}=-k T\left(\partial w_{/}^{\prime} \partial n\right)_{B},
$$

$S$ being the domain of the membrane.

We now make the transformation

$$
u=\frac{1}{2} r^{2}+2 T w / P,
$$

*Received Feb. 1, 1954. 